\title{
串列螺旋桨空化特性数值分析
}

杜晓旭，张正栋

(西北工业大学 航海学院, 陕西 西安 710072)

\begin{abstract}
摘 要: 运用计算流体力学 (CFD) 理论, 基于均质多相流雷诺平均 N-S (RANS) 方程, 结合 SST k- $\omega$ 湍 流模型及 Z-G-B (Zwart-Gerber-Belamri) 空化模型, 首先对 CLB4-55-1 串列螺旋桨的非空化定常水动力 特性及对 NACA66 翼型的定常流空化性能进行数值模拟,验证计算方法的可靠性; 然后对该串列螺旋 桨的空化性能展开数值模拟与分析。计算结果表明,所建立数值计算模型可以较为精确地预报串列 螺旋桨的空化性能; 串列桨的空化特性相较于单桨基本一致,但是在某些特定进速系数及空化数时, 后桨的空化现象较前桨更为明显; 当进速系数变大或空化数变大时,空化现象均会消失。
\end{abstract}

关 键 词: 串列螺旋桨; 空化特性;CFD; 混合网格; MATLAB; 网格划分; 湍流模型

中图分类号: U664.33

文献标志码: A

随着船舶主机功率的以及螺旋桨负载的增大， 螺旋桨空化、噪声问题更加突出,一种新型的螺旋 桨——串列螺旋桨进人人们的视野。串列螺旋桨是 一种 2 个普通螺旋桨位于同一转轴且转速及旋转方 向相同的特种推进器。与其他特种螺旋桨相比, 它 构造简单, 加工、安装及维修更加方便, 且当负载较 大时, 推进效率有所提高 ${ }^{[1]}$ 。串列螺旋桨在一段时 期并没有得到发展, 在 20 世纪 60 年代以后, 串列螺 旋桨才再次引起人们的兴趣, 国内外学者也做了一 定的研究 ${ }^{[1]}$ 。但总体来说, 有关串列螺旋桨方面的 研究较少, 有关串列螺旋桨空化的研究更是少之又 少, 因此本文研究将对串列螺旋桨的发展奠定一定 的基础。

对于螺旋桨空化特性的研究, 主要有实验和数 值模拟 2 种方法, 前者耗时耗力且需要一定的实验 条件, 而随着计算机技术的发展, 计算流体力学 (CFD) 技术蓬勃发展, 基于数值模拟技术进行科学 研究已经成为现在的研究主流, 而且其具有信息量 全且容易实现的特点, 深受研究者们的喜爱。基于 势流理论及黏流理论, 利用面元法及求解 RANS 方 程来数值模拟螺旋桨水动力特性及空化特性的技术 已经日渐成熟, 且通过研究者们进行对比, 数值计算 结果相当可靠, 目前国内外已经取得了很多相关成
文章编号 : 1000-2758(2018)03-0509-07
果 ${ }^{[4]}$ 。对于串列螺旋桨水动力特性, 余欣 ${ }^{[7]}$ 基于 CFD 方法对串列螺旋桨的水动力性能展开较为系 统的研究; 王超等人 ${ }^{[8]}$ 通过求解 RANS 方程对串列 螺旋桨水动力性能展开数值预报, 并与试验结果进 行对比,发现该方法计算结果与试验结果吻合。

对于串列螺旋桨空化方面的研究, 目前并没有 相关的论文, 考虑到 Ahn 等人 ${ }^{[9]}$ 基于非结构化网 格, 通过求解多相流 RANS 方程计算了 P4381 型螺 旋桨的定常流空化特性, 发现数值计算结果能够很 好地预报空化的尺寸及形状等, 认为通过数值模拟 方法分析串列螺旋桨空化特性是可行的。故本文基 于黏性流理论及多相流理论, 结合剪切应力 SST k$\omega$ 湍流模型及 Z-G-B (Zwart-Gerber-Belamri) 空化模 型, 通过求解 RANS 方程来求解三维全通道串列螺 旋桨的定常空化流场, 通过研究不同进速系数及不 同空化数下串列螺旋桨的性能, 为串列螺旋桨的设 计和研究提供一定的参考。

\section{1 数值方法}

\section{1 控制方程}

在数值分析螺旋桨空化特性时需考虑汽液两相 
混合流模型, 该模型考虑了流场在相变过程中两相 间的相互影响以及滑移速度, 通过相变率引人空化 模型。当发生空化时, 空化流体通常表示为水及水 蒸汽的混合物, 因此空化流场表示为密度可变的统 一混合流场。在这里引人汽相传输方程来求解汽相 体积分数, 可以得到混合密度 $\rho_{m}$ 与空泡相质量分数 $f_{v}$ 的关系如下:

$$
\frac{1}{\rho_{m}}=\frac{f_{v}}{\rho_{v}}+\frac{1-f_{v}}{\rho_{l}}
$$

因此混合流的控制方程 ${ }^{[10]}$ 在直角坐标系下的张量 形式如下:

$$
\begin{aligned}
& \frac{\partial}{\partial t}\left(\rho_{m}\right)+\frac{\partial}{\partial x_{j}}\left(\rho_{m} u_{j}\right)=0 \\
& \frac{\partial\left(\rho_{m} u_{i}\right)}{\partial t}+\frac{\partial}{\partial x_{j}}\left(\rho_{m} u_{i} u_{j}\right)=-\frac{\partial p}{\partial x_{i}}+ \\
& \frac{\partial}{\partial x_{j}}\left[\left(\mu+\mu_{m}\right)\left(\frac{\partial}{\partial x_{j}} u_{i}+\frac{\partial}{\partial x_{j i}} u_{j}\right)\right]
\end{aligned}
$$

式中, $i, j=1,2,3 ; \rho_{v}$ 为空泡相密度; $\rho_{l}$ 为液体相密 度 $; u_{i}, u_{j}$ 为混合相速度; $x_{i}, x_{j}$ 为空间坐标分量 $; \mu_{m}$ 为 混合黏性系数。

\section{2 空化模型}

相较于应用最广泛的 Singhal 空化模型, Z-G-B 空化模型 ${ }^{[11]}$ 能够更好地模拟发生空化时空泡从出 现到增大的过程, 且在传质速率相中考虑了非凝结 气体 $(\mathrm{NCG})$ 体积分数的影响, 因此本文选用 Z-G-B 空化模型。其控制空泡体积分数 $\phi_{v}$ 的方程定义为:

$$
\frac{\partial\left(\phi_{v} \rho_{v} u_{j}\right)}{\partial\left(x_{j}\right)}=\dot{m}^{+}-\dot{m}^{-}
$$

式中, $\dot{m}^{+}$和 $\dot{m}^{-}$分别为空泡的生成和凝结速率, 两 者都为压力、速度以及流体密度等参数的结合函数, 将 $\dot{m}^{+}$中 $\phi_{v}$ 换为 $\phi_{\mathrm{NCG}}\left(1-\phi_{v}\right)$, 则表达式定义为:

$$
\begin{gathered}
\dot{m}^{+}=F_{\text {vap }} \frac{3 \phi_{\mathrm{NCG}}\left(1-\phi_{v}\right) \rho_{v}}{R_{B}} \sqrt{\frac{2}{3} \frac{\left|p_{v}-p_{s}\right|}{\rho_{l}}} \\
\dot{m}^{-}=F_{\text {cond }} \frac{3 \phi_{v} \rho_{v}}{R_{B}} \sqrt{\frac{2}{3} \frac{\left|p_{v}-p_{s}\right|}{\rho_{l}}}
\end{gathered}
$$

式中, $F_{\text {vap }}$ 和 $F_{\text {cond }}$ 分别是空泡衍生和凝结的经验系 数; $R_{B}$ 是空泡半径; $\phi_{\mathrm{NCG}}$ 为 $\mathrm{NCG}$ 体积分数; $p_{v}$ 为空泡 内压强; $p_{s}$ 为空泡周围压强。

SST k- $\omega$ 湍流模型 ${ }^{[12]}$ 能够更加精确地预报存在 负压时流体的分离量以及更好地处理不同边界层问 题, 在计算复杂螺旋桨流场时有更广泛的适用性, 故 本文选用 SST k- $\omega$ 湍流模型来封闭 RANS 方程。

\section{2 计算模型与网格划分}

\section{1 几何模型}

本文以标准 CLB4-55-1 串列螺旋桨 ${ }^{[13]}$ 为仿真 模型, 其主要参数如表 1 所示。

\section{表 1 CLB4-55-1 串列螺旋桨几何参数}

\begin{tabular}{ccc}
\hline 参数 & 前桨 & 后桨 \\
\hline 叶型 & \multicolumn{2}{c}{ B 型 } \\
盘面比 & \multicolumn{2}{c}{0.55} \\
叶数 $Z$ & \multicolumn{2}{c}{4} \\
直径 $D / \mathrm{mm}$ & 220 & 220 \\
厚度比 $t / D$ & 0.045 & 0.045 \\
壳径比 $d / D$ & 0.182 & 0.182 \\
螺距比 $P / D$ & 1.1 & 1.2 \\
桨距比 $L / D$ & 0.21 & \\
叶错角 $\theta /\left(^{\circ}\right)$ & 23.7 & \\
\hline
\end{tabular}

桨模叶剖面和叶轮廓与荷兰 Wageninger 水池 的 B 系列相同, 有关型值点见文献 [13], 但螺距径 向不变,且无后倾角。

与普通螺旋桨相似, 模型建立于直角坐标系。 $Y$ 轴为旋转轴, 指向来流方向; $X$ 轴为桨叶的叶面母 线方向, 由叶根指向叶梢; $Z$ 轴负荷右手螺旋定则。 通过坐标转换, 将二维型值点文件通过 MATLAB 程 序转化到所建三维坐标系, 对叶梢与叶根做适当处 理。为了更好地模拟来流, 在桨觳前后端添加半球 体导流帽与尾流罩。同时, 为了提高数值计算的精 度, 采用全通道模型展开数值计算, 几何模型如图 1 所示, 红色桨叶为后桨。

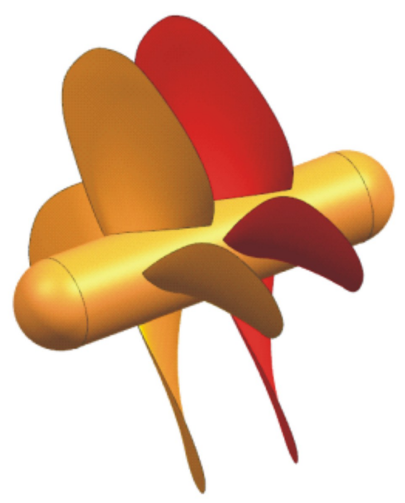

图 1 串列螺旋桨几何模型 


\section{2 网格划分}

网格划分直接影响数值计算精度与速度, 划分 高质量的网格是数值计算必须满足的条件。鉴于本 文所计算的旋转模型, 采用分块混合网格的方法划 分高质量网格。将计算域分为静止域与旋转域两部 分, 旋转域包含螺旋桨, 为旋转计算域。2 个域通过 交界面来传输数据。由于交界面之间存在滑移, 故 通过滑移网格技术来模拟 2 个域之间的相互作用。

计算域为半径 $5 D$, 长 $10 D$ 的圆柱体, 人口距螺 旋桨 $4 D$ 。静止域采用六面体结构化网格, 旋转域采 用非结构化四面体网格, 采用分区域加密的手段在 螺旋桨周围加密网格, 在螺旋桨叶面划分 5 层三棱 柱边界层网格, 第一层网格高度约为 $0.0001 D$, 总体 网格数约为 280 万。计算域及网格如图 2 、图 3 所 示所示。

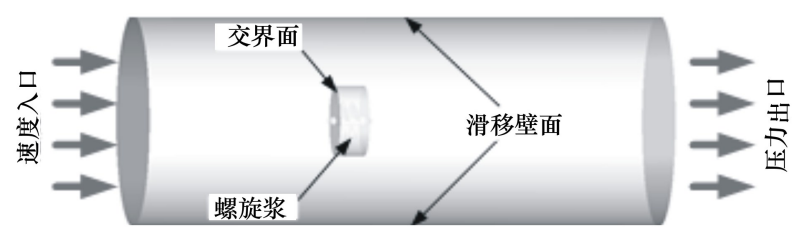

图 2 计算域及边界条件

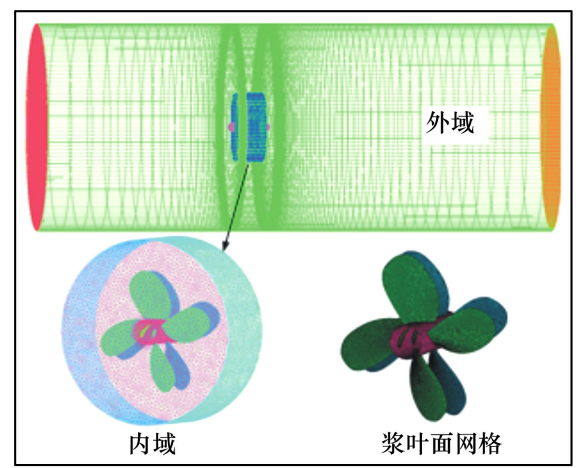

图 3 网格划分

\section{3 计算方法与边界条件}

本文采用有限体积法 (FVM) 来离散 RANS 方 程, 采用高精度差分格式进行空间离散, 采用 Euler 后插格式进行时间离散。人口边界为速度人口, 湍 流强度为 $5 \%$, 液体的体积分数为 1 , 空泡的体积分 数为 0 , 液体温度 $25^{\circ}$, 饱和蒸汽压为 $3540 \mathrm{~Pa}$, 空泡 的平均直径为 $2 \mu \mathrm{m}$, 出口边界为压力出口, 由空化 数 $\sigma=\left(p_{\text {out }}-p_{v}\right) / 0.5 \rho_{l} V^{2}$ 进行控制, $V$ 为来流速度, 螺旋桨壁面为无滑移壁面,近壁区域采用增强壁面
函数模型, 在圆柱壁面采用自由滑移壁面条件。本 文通过结合全隐式耦合及并行计算技术来提高技术 速度与稳定性, 收玫条件为 $10 \times 10^{-5}$ 。

\section{3 数值计算}

为了方便对计算结果的处理与分析对比,特对 相关物理参数进行无量纲化处理: 进速系数 $J=$ $V / n D$, 串列桨推力系数为 $K_{T}=\left(T_{1}+T_{2}\right) / \rho n^{2} D^{4}$, 扭 矩系数 $K_{Q}=\left(Q_{1}+Q_{2}\right) / \rho n^{2} D^{4}$, 敞水效率为 $\eta=$ $K_{T} / K_{Q} *(J / 2 \pi)$ 。其中: $n$ 为螺旋桨转速, 在本文取 $900 \mathrm{r} / \mathrm{min}, T_{1}$ 与 $T_{2}$ 分别为前后桨推力, $Q_{1}$ 与 $Q_{2}$ 分别 为前后桨扭矩。

\section{1 水动力特性及空化特性验证}

为了验证本文所建立几何模型及定常非空化流 场数学模型的精确性, 本文首先对数值计算的网格 无关性进行验证, 通过调节网格尺度来改变网格数 目,表 2 所示为不同网格数目下 CLB4-55-1 串列螺 旋桨推力系数, 可以看出当网格数目达到 280 万时, 计算结果已经几乎保持不变, 故选择 280 万网格作 为全文计算网格。基于网格无关性测试, 对进速系 数 $J$ 从 0.4 1.1 变化的 CLB4-55-1 串列螺旋桨水动 力系数进行计算, 并与实验值进行对比, 对比结果如 图 4 所示。

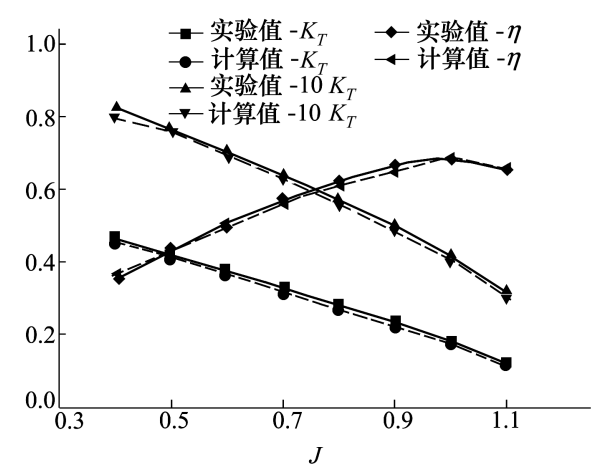

图 4 水动力特性对比曲线

可以看出,推力系数 $K_{T}$ 与 10 倍扭矩系数 $10 K_{Q}$ 与实验值基本吻合, 当进速系数为 0.4 时, $K_{T}$ 与实 验值的误差最大, 为 0.023 , 误差约为 $2.87 \%$, 故本文 所建立非空化计算模型计算结果是可靠的。

为了验证本文所建立空化模型的准确性, 特选 取二维 NACA66 翼型作为算例进行空化验证。利用 ANSYS FLUENT 软件展开定常空化性能数值计算。 
计算网格及边界条件设置如图 5 所示。

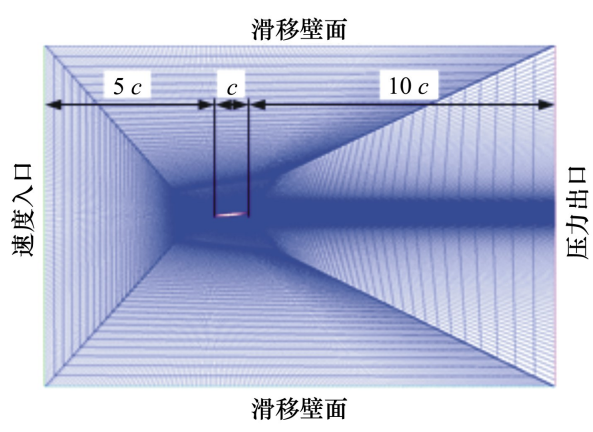

图 5 NACA66 翼型网格划分及边界条件设置

翼型弦长 $c$ 为 $100 \mathrm{~mm}$, 翼型攻角 $\alpha=2^{\circ}$, 翼型表 面为无滑移壁面。计算设置时, 选用液态水及水蒸 汽, 密度分别为 $998.2 \mathrm{~kg} / \mathrm{m}^{3}$ 及 $0.0019 \mathrm{~kg} / \mathrm{m}^{3}$, 温度 为 $25^{\circ} \mathrm{C}$, 饱和蒸汽压为 $3540 \mathrm{~Pa}$, 来流速度设为 10 $\mathrm{m} / \mathrm{s}$, 通过改变出口压来调节空化数 $\sigma_{\text {。 }}$

表 2 网格无关性测试

\begin{tabular}{|c|c|c|c|c|c|}
\hline 网格数目/万 & 180 & 230 & 280 & 310 & 350 \\
\hline$K_{T}$ & $0.431 c$ & 0.445 & 0.457 & 0.4580 & 0.4581 \\
\hline
\end{tabular}

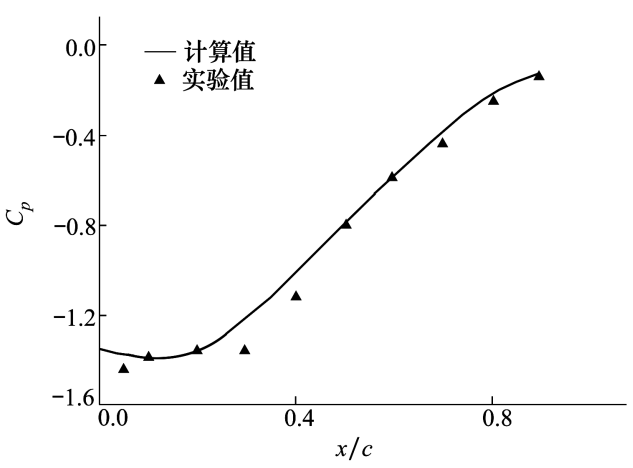

a) $\sigma=1.29$

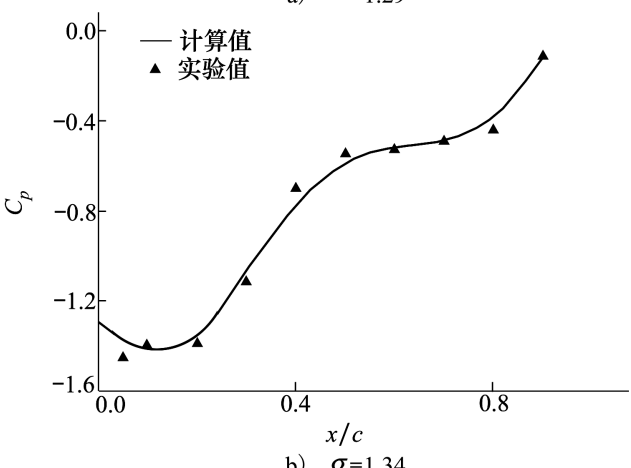

b) $\sigma=1.34$

图 6 数值计算结果与实验结果对比

图 6 为 2 种最为经典的空化数 $\sigma$ 对应的翼下表
面的压力系数弦向分布图, 压力系数 $C_{p}=(p-$ $\left.p_{\text {out }}\right) / 1 / 2 \rho_{l} V^{2}$, 图中 $x$ 为压力点位置与导边的距离。 由图可知, 本文数值计算结果除了导边处其他地方 与实验值 ${ }^{[10]}$ 基本一致, 通过上述算例计算结果对比 实验验证了本文空化模型的精确性及所选择数值计 算方法的可靠性, 从而保证了后续计算结果的可 靠性。

\section{2 不同进速系数时计算结果分析}

为了研究不同进速系数对串列螺旋桨空化特性 的影响, 本文通过调节来流速度来改变进速系数, 本 文进速系数变化范围为 $0.6 \sim 1.2$ 。

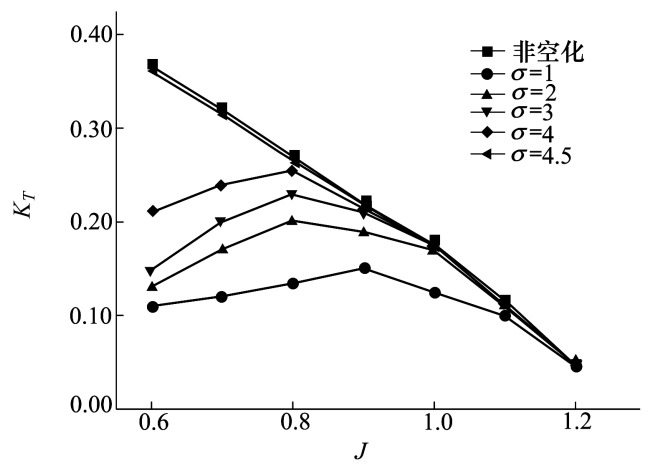

图 7 不同空化数敞水推力特性曲线

图 7 所示为不同空化数对应的串列螺旋桨敞水 推力特性曲线与敞水非空化推力特性曲线对比图, 可以看出, 空化数越小, 进速系数越小, 空化现象越 明显, 导致螺旋桨推力越小。当 $\sigma=1$ 时,在所有进 速系数范围内, 螺旋桨均发生空化, 故推力系数整体 低于非空化推力系数, 随着进速系数增大, 推力系数 先增加后减小; 当 $\sigma=2$ 时, 进速系数小于 0.7 时, 空 化面积很大, 推力系数与 $\sigma=1$ 时的推力系数相差不 大。当进速系数大于 0.7 小于 1 时, 桨叶局部空化, 推力系数有所增加, 当进速系数大于 1 时, 桨叶不发 生空化, 推力系数与非空化推力系数非常接近; 当 $\sigma$ $=3$ 时, 低进速系数时, 桨叶出现局部非空化, 因此推 力系数相比于低空化数时有所增加; 当 $\sigma=4$ 时, 由 于空化数较大, 因此发生空化面积较小, 故当进速系 数大于 0.9 时, 已不再产生空化现象, 推力系数也无 变化。当 $\sigma=4.5$ 时,已经不再发生空化, 敞水推力 特性与非空化推力特性十分接近。总之, 空化数越 大, 出口压力越大, 液态水的汽化压力与当地流体压 力的差值越来越大, 因此空化现象逐渐消失。

图 8 为 2 个不同空化数 $\sigma=2$ 及 $\sigma=3$ 时桨叶的 
吸力面空化云图。空化数 $\sigma=2$ 时, 可以清楚地看出 随着进速系数的增大, 空化区域减小。当进速系数 为 0.6 时, 前桨吸力面已经完全空化, 后梁除了靠近 桨毂的区域外, 其他地方也完全空化, 该进速系数空 化现象明显。当进速系数为 0.8 时, 前桨与后桨都 为局部空化,这也与图 7 敞水推力系数变化曲线向 对应。需要提到的是, 当进速系数为 0.9 时, 后桨空 化区域比前桨空化区域更大, 可能有 2 个原因: (1) 后 桨螺距较前桨大 0.1 , 空化性能差于前桨; (2) 前桨尾 流场对后桨由影响, 在后桨空化区域出现回流涡, 流 体速度增大, 压强减小, 发生空化。当进速系数大于 0.9 时, 已经不再发生空化。空化数 $\sigma=3$ 时, 空化现 象明显有所抑制, 当进速系数为 0.6 时, 前桨和后桨 都为局部空化。当进速系数为 0.8 时, 前桨已经不 发生空化, 而后桨在叶梢附近还有局部空化。当进 速系数大于 0.8 时, 不发生空化。对比同一个进速 系数不同空化数的桨叶空化图可发现, 空化数越小, 空化区域越大,空化现象越明显。

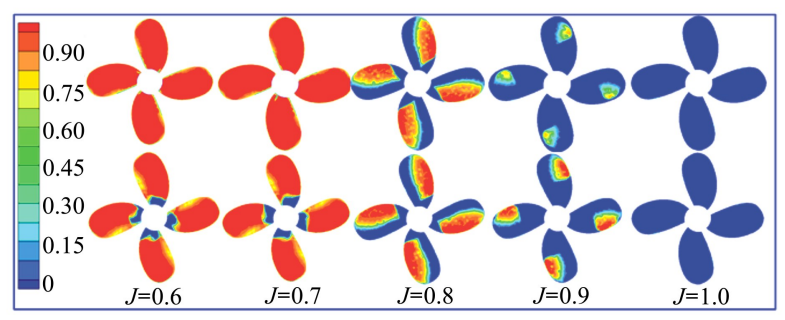

a) $\sigma=2$ 时吸力面空化图 (上方为前浆, 下方为后浆)

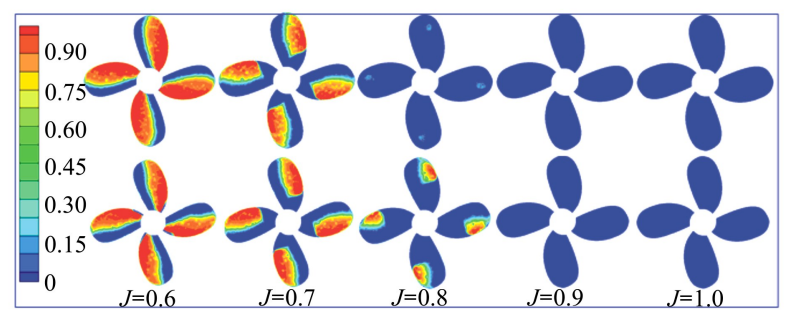

b) $\sigma=3$ 时吸力面空化图 (上方为前浆,下方为后浆)

图 8 不同进速系数时吸力面空化云图

\section{3 不同空化数时计算结果分析}

图 9 所示为空化数在 1 4.5 变化的串列螺旋桨 敞水效率曲线, 由图可以看出, 对于固定的进速系 数, 随着空化数的增加, 效率呈现先增加后基本不变 的趋势, 分析其原因, 当空化数小时, 空化现象尤为 明显, 前桨及后桨的吸力面几乎都被气体覆盖, 导致 效率有很大的损失, 而随着空化数增加, 空化现象消 失, 因此效率逐渐保持不变。进速系数越大, 效率损
失越少, 进速系数是有由来流速度决定的, 来流速度 越大, 对于固定空化数, 出口压力也越大, 同时桨叶 部分的动压越大, 因此液态水的汽化压力与叶片部 分流体压力的差值越大, 空化现象被抑制, 甚至当进 速系数为 1.1 时, 基本不发生空化。当进速系数为 0.7 时,效率损失尤为验证,近乎 $20 \%$, 由图 7 可知, 此时推力损失较大, 故导致效率损失较大。

图 10 为进速系数为 0.9 时不同空化数时的桨 叶空化云图, 可以看出随着空化数的增加, 空化现象 得到抑制, 最后消失。当空化数 $\sigma=1$ 时, 前桨除了 叶根靠近桨觳处没有空化, 其他部位都发生空化, 而 后桨有几乎 $1 / 3$ 的叶面积为发生空化, 区域为靠近 桨毂处, 这是由于靠近桨毂处的流体速度较低造成 的。而当空化数 $\sigma=1.5$ 时, 空化区域随着流体运动 方向向桨叶随边处移动, 前后桨发生空化区域几乎 一致,而当空化数 $\sigma=2$ 时, 前桨空化区域几乎消失, 只剩一小部分区域发生空化, 而后桨由于前桨尾流 场的加速作用, 还有较大区域发生空化。当空化数 $\sigma>2$ 时,空化现象消失, 可见对于进速系数为 0.9 , 该型串列螺旋桨不再发生空化。

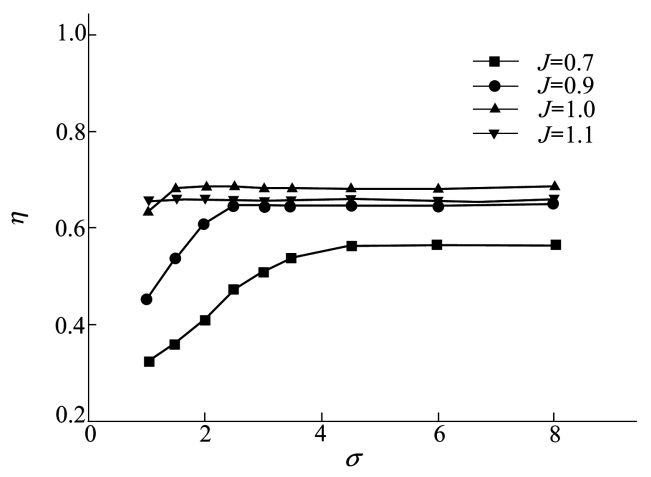

图 9 不同空化数时敞水效率

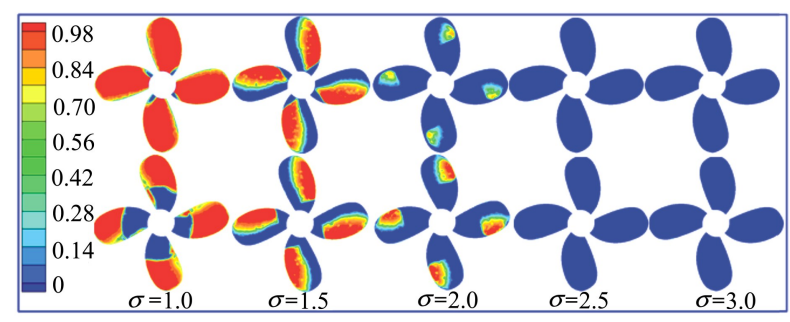

图 10 不同空化数吸力面空化云图 (上为前桨,下为后桨) 


\section{4 结 论}

本文基于混合网格的 RANS 方法来分析和预报 了 CLB4-55-1 型串列螺旋桨在均匀来流情况下的空 化性能, 在完成该桨水动力特性及经典空化算例验 证的基础上,对不同进速系数及不同空化数下的串 列螺旋桨空化性能展开数值预报, 通过分析得到以 下结论:

1) 对 CLB4-55-1 螺旋桨进行非空化敞水定常水 动力计算, 计算结果与实验结果一致, 验证了本文所 建立几何模型及网格划分的准确性与可靠性; 对 NACA66 翼型进行定常空化性能数值模拟, 对于不 同空化数下的翼型压力分布于实验结果基本一致, 验证了所建立空化模型的合理性与实用性。

2) 随着进速系数的升高, 螺旋桨空化情况下敞 水推力系数呈降低趋势; 空化数越大, 空化推力系数
与非空化推力系数之间的差越小; 进速系数越小, 空 化现象越明显; 当进速系数为 1 时,非空化推进效率 最大, 当进速系数大于 1 时且空化数大于 1 时, 几乎 不发生空化,对空化效率无较大影响。

3 ) 随着空化数的增大, 空化现象逐渐被抑制, 最后空化消失; 发生空化时, 敞水推进效率有明显下 降, 进速系数越小, 空化越明显, 效率下降越多; 随着 空化消失, 推进效率逐渐基本不变; 当进速系数为 0.9 时, 空化数小于 2 时, 空化现象明显, 大于 2 时不 发生空化。

4) 串列桨空化特性与单桨基本一致,在低进速 系数及低空化数时, 前后桨吸力面均发生大面积空 化,但当进速系数增大到 0.8 时,空化现象逐渐被抑 制, 此时由于前桨对流场的加速及回流涡的存在, 后 桨空化面积略大于前桨空化面积, 当空化数达到某 一值时,前后桨空化现象消失。

\section{参考文献:}

[1] 盛振邦, 刘应中. 船舶原理 $[M]$. 上海: 上海交通大学出版社, 2004:196-197

Sheng Zhenbang, Liu Yingzhong. Ship Theory [M]. Shanghai, Shanghai Jiaotong University Press, 2004: 196-197 (in Chinese)

［2］孙勤，顾蕴德，郑淑珍. 串列螺旋桨的模型系列实验和图谱设计方法 [J]. 中国造船，1979，20(3): 3-20

Sun Qin, Gu Yunde, Zheng Shuzhen. On the Open Water Series Test of Model Tandem-Propeller and its Design Method with Charts $[\mathrm{J}]$. Shipbuilding of China, 1979, 20(3): 3-20 (in Chinese)

[3] Titoff I A, Biskoup B A. Investigation into the Possibilities of Tandem Propeller Application with the Aim of Decreasing the Variable Hydrodynamic Loads Transmitted to a Propeller Shaft $[$ C ] // Proceedings of the 11th International Towing Tank Conference. Tokyo, Japan, 1966: 308-314

[4] 郑小龙, 黄胜, 王超. 基于 CFD 的螺旋桨定常水动力性能预报精度研究 [ J ]. 舰船科学技术, 2014, 36(12)：11-15 Zheng Xiaolong, Huang Sheng, Wang Chao. Study of Precision of Steady Hydrodynamic Performance Prediction of Propeller of Based on CFD $[\mathrm{J}]$. Ship Science And Technology, 2014, 36(12): 11-15 (in Chinese)

[5] Rhee S H, Kawamura T, Li H. Propeller Cavitation Study Using an Unstructured Grid Based Navier-Stoker Solver[J]. Journal of Fluids Engineering, 2005, 127( 5 ) :986-994

[6] Pereira F A, Di Felice F, Salvatore F. Propeller Cavitation in Non-Uniform Flow and Correlation with the Near Pressure Field [J]. Journal of Marine Science and Engineering, 2016, 4(4):70

[7] 余欣. 基于 CFD 方法的串列螺旋桨水动力性能研究 [D]. 大连:大连理工大学, 2014

Yu Xin. The Research on the Hydrodynamic Performance of Tandem Propellers Based on CFD Methods[J]. Dalian, Dalian University of Technology, 2014 ( in Chinese)

[8] 王国亮，王超，乔越,等. 串列螺旋桨水动力性能的数值预报 $[\mathrm{J}]$. 舰船科学技术, 2016, 38(3): 10-13

Wang Guoliang, Wang Chao, Qiao Yue, et al. Numerical Prediction of the Propeller's Hydrodynamics Performance[J]. Ship Science and Technology, 2016, 38(3): 10-13 (in Chinese)

[9] Ahn S J, Kwon O J. Numerical Investigation of Cavitating Flows for Marine Propellers Using an Unstructured Mesh Technique [J]. International Journal of Heat and Fluid Flow, 2013, 43( 5) : 259-267

[10] 王献孚. 空化泡和超空化泡流动理论及应用 $[M]$. 北京: 国防工业出版社, 2009 
Wang Xianfu. Cavitating and Supercavitating Flows Theory and Applications [M]. Beijing, National Defense Industry Press, 2009 (in Chinese)

[11] Ji B, Luo X W, Wang X, et al. Unsteady Numerical Simulation of Cavitating Turbulent Flow around a Highly Skewed Model Marine Propeller $[\mathrm{J}]$. Journal of Fluids Engineering, 2011, 133( 1) : 21-28

[12] Ji B, Luo X W, Wu Y L, et al. Numerical Investigation of Unsteady Cavitating Turbulent Flow around a Full Scale Marine Propeller [J]. Journal of Hydrodynamics, 2010, 22( 5) : 747-752

[13] 孙勤. 串列螺旋桨及其设计方法 $[M]$. 北京: 人民交通出版社, 1983

Sun Qin. Tandem Propeller and Design Method[M]. Beijing, China Communications Press, 1983 (in Chinese)

[14] Liu Yan, Zhan P F, Wang Q, et al. Urans Computation of Cavitating Flows around Skewed Propellers[J]. Journal of Hydrodynamics, 2012, 24(3):339-346

\title{
Numerical Simulation Analysis of Cavitation Performance of Tandem Propeller
}

\section{Du Xiaoxu, Zhang Zhengdong}

( School of Marine Science and Technology, Northwestern Polytechnical University, Xi' an 710072, China)

\begin{abstract}
The steady non cavitation hydrodynamic characteristics of CLB4-55-1 tandem propeller and the steady cavitation flows of NACA66 hydrofoil are numerically studied firstly based on the RANS equations of homogeneous multiphase using CFD theory, combined with the SST k- $\omega$ turbulent model and Z-G-B cavitation model. Numerical simulation results are in good agreement with the experimental results, which indicates that the numerical method is reliable and accurate. Then, the cavitation performance of the tandem propeller are numerical simulated and analyzed. The results show that the computational model can predict the cavitation performance of tandem propeller accurately. The cavitation performance of tandem propeller is nearly the same as single propeller, however, the cavitation phenomenon of back propeller is greater than the head propeller at certain advance coefficient and cavitation number. The cavitation phenomenon will disappear with the increase of the advance coefficient or the cavitation number.
\end{abstract}

Keywords: tandem propeller; cavitation performance; CFD; hybrid mesh; MATALB; mesh generation; turbulent model 